\title{
Advantages of HAADF vs. Conventional TEM Imaging for Study of PSS-PMB Diblock Copolymer Systems
}

\author{
S. Yakovlev,* K, H Downing, ${ }^{*}$ X. Wang, ${ }^{* *}$ and N. Balsara ** \\ * Lawrence Berkeley National Laboratory, Berkeley, CA 94720 \\ ** University of California, Berkeley, CA 94710
}

It is well known that HAADF STEM imaging provides much stronger selectivity for heavy atoms than Conventional Bright Field TEM (CTEM) imaging. For some materials such selectivity may provide a significant advantage and allow collection of higher resolution images that may be easily interpreted. Another less known advantage of HAADF imaging is that radiation damage may be manifested differently compared to CTEM. In this work we examine the difference between HAADF and CTEM imaging using as an example the sulfonated polystyrene/polymethylbutene diblock copolymer. This material shows strong phase separation [1]. The polymethylbutene phase consists of carbon and hydrogen atoms bonded by single bonds while polystyrene also has an aromatic ring with double bonds and a sulfuric acid group. While the sulfur content of this material is below 1 atomic percent, it is enough to provide a substantial contribution to the high angle scattering. In contrast, low angle scattering is dominated by the density variations due to the presence of the more compact aromatic ring in polystyrene. We demonstrate that TEM illumination causes rapid damage and disappearance of the observed in TEM morphology while HAADF images are much less affected by irradiation damage.

Figure 1a presents CTEM images collected from PSS-PMB sample using a dose of $10^{3} \mathrm{e} / \mathrm{nm}^{2}$. The image clearly shows phase separation in the material. Figure $1 \mathrm{~b}$ presents an image collected from the same area of the sample after irradiation with $10^{4} \mathrm{e} / \mathrm{nm}^{2}$. One can see that such irradiation destroys sample morphology, and the phase separation can hardly be detected. However, the HAADF image collected from the same area (Figure 1c) clearly reveals that the sample is not uniform and the initial morphology is preserved even though it is not detectable in the bright field image. To explain this behavior we suggest that irradiation damage destroys some of the double bonds [2] and at the same time creates new double bonds in both polystyrene and polymethylbutene. In the end the polystyrene density becomes equal to the density of the polymethylbutene. This process relies on irradiation induced diffusion of hydrogen [3] which redistributes hydrogen and as a consequence redistributes double bonds in the material. In contrast to hydrogen sulfur, being a heavier atom, does not diffuse into different phase. At the same time sulfur does not significantly influence the density of polystyrene due to its low concentration. When the density of the phases becomes equal contrast in bright field disappears. This process however does not significantly affect the HAADF image because the concentration of the sulfur responsible for image contrast does not change.

The ability to apply higher doses coupled with the fact that HAADF imaging does not require any defocus allows us to collect images with resolution better than $1 \mathrm{~nm}$, which would not be possible using CTEM. Figure 2 presents high resolution HAADF image together with component and thickness maps derived from the low loss EELS spectrum image with the pixel size $3 \mathrm{~nm}$ and collected from the same area. One can clearly see correlation between the component map and HAADF image while the thickness only shows mild changes not correlated with HAADF image. These maps support the suggestion that the HAADF image is dominated by elemental composition 
rather than density variations. At the same time the resolution of the HAADF image is significantly higher than the resolution of the sulfur map. Based on the simple element composition of the sample we conclude that the HAADF image can be treated as a high resolution sulfur map. We found further support for this notion by collecting a sulfur L edge $(165 \mathrm{eV})$ map. While the sulfur map obtained has a high amount of noise we were able to observe correlation between the sulfur map and HAADF image.

Analyzing high resolution HAADF images we can draw important conclusion about morphology of the material. The most important observation is that sulfur is distributed nonuniformly in polystyrene creating clusters similar to the clusters detected by scattering experiments in the homopolymer Nafion. Clustering of sulfur must have a big impact on such properties of polymer as conductivity and water permeability. HAADF imaging is a most convenient method to study the morphology dependence of such properties.

\section{References}

[1]. Park, M.J., et al., Control of domain orientation in block copolymer electrolyte membranes at the interface with humid air. Advanced Materials, 2009. 21(2): p. 203-208.

[2]. Varlot, K., J.M. Martin, and C. Quet, Physical and chemical changes in polystyrene during electron irradiation using EELS in the TEM: Contribution of the dielectric function. Journal of Microscopy, 1998. 191(2): p. 187194.

[3]. Yakovlev, S., et al., Specimen thickness dependence of hydrogen evolution during cryo-transmission electron microscopy of hydrated soft materials. Journal of Microscopy, 2009. 236(3): p. 174-179.

[4]. Authors are grateful to Dr. R. Glaeser of Lawrence Berkeley National Laboratory for useful discussion during the work on this project. This work was supported by the Office of Science, Office of Basic Energy Sciences, of the U.S. Department of Energy under Contract No. DE-AC02-05CH11231.
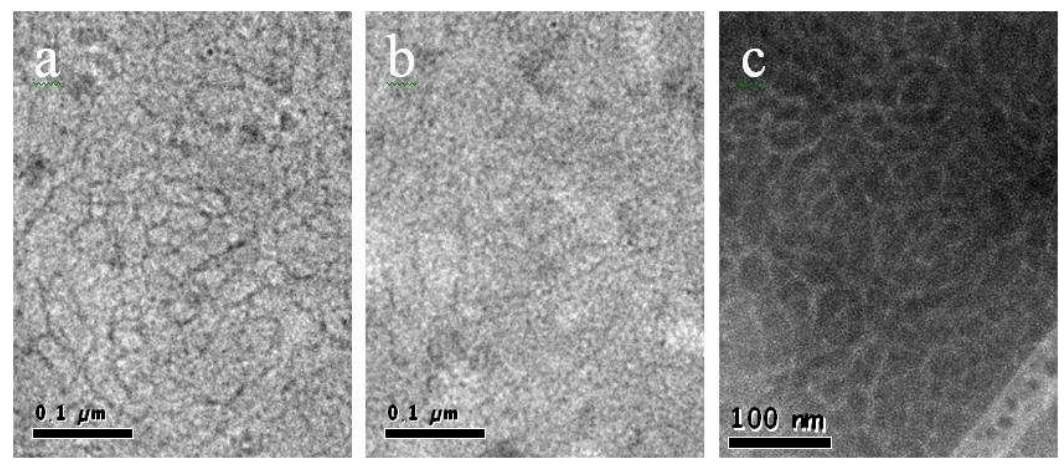

FIG. 1. a) TEM image of undamaged PSS/PMB film, b) TEM image of PSS/PMB film damaged with $10^{4} \mathrm{e} / \mathrm{nm}^{2}$ irradiation, c) HAADF image of damaged PSS-PMB film. Images $a$ and $b$ collected under exactly the same condition from the same area of specimen. For collection of all images same irradiation dose was used.
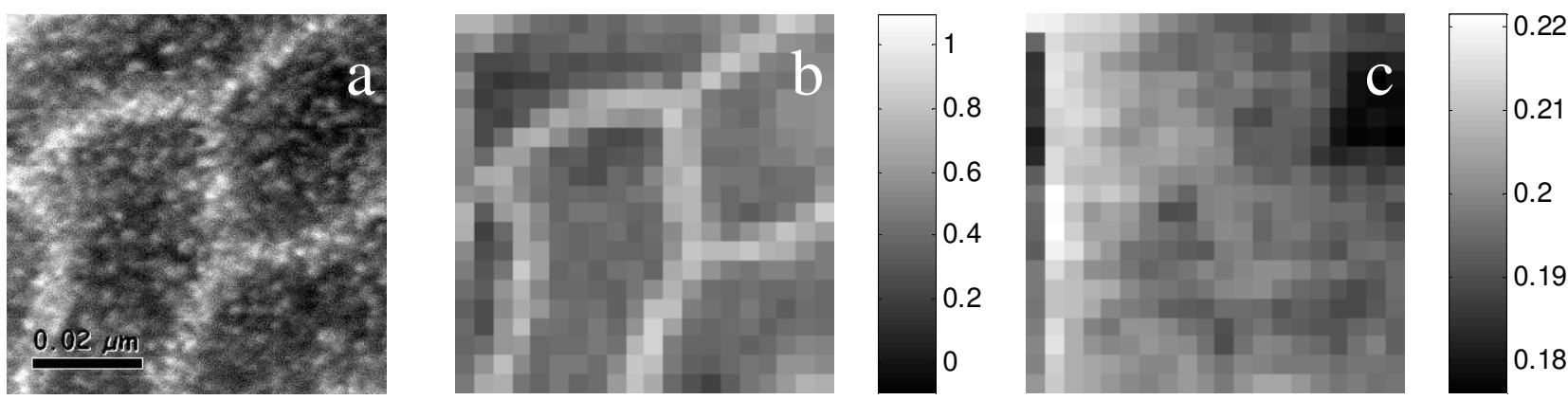

FIG. 2. a) High resolution HAADF image, b) component map of the same area as image a, c) thickness map of the same area 